\title{
Dermatofibrosarcoma protuberans of the breast: A rare diagnosis requiring aggressive initial surgical therapy
}

Navin Sookar, Jacinta Bisnath, Jameel Ali

Department of Women's Health, St. James Medical Complex, Trinidad and Tobago

Received: July 30, 2018

DOI: $10.5430 /$ css.v4n2p25
Accepted: September 13, 2018 Online Published: September 18, 2018

URL: https://doi.org/10.5430/css.v4n2p25

\begin{abstract}
Dermatofibrosarcoma protuberans (DFSP) is an extremely rare cutaneous neoplasm of the dermal layer of the skin and is histologically classified as a sarcoma. DFSP itself has an incidence of 0.8 cases per million annually. It accounts for $1 \%$ of all soft tissue sarcomas and less than $0.1 \%$ of all malignancies. DFSP has a high rate of local recurrence especially if there are positive margins on excision, but only up to $4 \%$ develop metastasis. Most reported cases are located in the trunk, extremities and head especially scalp. However, DFSP of the breast is very rare with very few reported in the literature. We report a case of a 33-year-old woman who presented with a histological diagnosis of DFSP of the breast, based on incisional biopsy. We report this case which highlights the important aspects in evaluating DFSP in the breast as well as its treatment.
\end{abstract}

Key Words: Dermatofibrosarcoma protuberans

\section{INTRODUCTION}

Dermatofibrosarcoma protuberans (DFSP) is a rare tumour of the subcutaneous tissue with intermediate malignant potential that rarely metastasizes. ${ }^{[1,2]}$ If metastasis does occur, they usually present several years after the initial presentation. ${ }^{[3-5]}$ The trunk and proximal limbs are the most common locations, but it has been reported in other sites. Very few cases of DFSP of the breast have been reported in modern literature. ${ }^{[6]}$ A review of the literature suggests that DFSP mainly occurs between the second and fifth decades of life. ${ }^{[7]}$

We highlight a case of DFSP in the breast which was diagnosed on incisional biopsy. Both clinical examination and imaging ruled out any other lesions in either breasts. Our case of locally invasive DFSP was initially treated with wide local excision. She underwent re-excision due to positive margins. She opted not to undergo radiotherapy. She has since been 3 years disease free with no signs of local or distant recurrence.

Early diagnosis and surgery with the goal of clear margins remains the mainstay of treatment of patients with DFSP. Due to its high recurrence rate, close follow-up and monitoring is also advised. ${ }^{[8]}$

\section{Case presentation}

A 33-year-old previously well female with no significant comorbidities presented to her private family physician with a six-month history of a painful non-enlarging skin lesion over the inferior left breast. It was irregularly demarcated, firm in

\footnotetext{
*Correspondence: Navin Sookar; Email: navin_shawn@hotmail.com; Address: Department of Women’s Health, St. James Medical Complex, 112 Western Main Road, St. James, Trinidad and Tobago.
} 
consistency and tan-brown in colour. She was then referred for an ultrasonogram of both breasts which demonstrated an area of skin thickening of $1.6 \mathrm{~cm} \times 0.3 \mathrm{~cm}$ area in the 7 o'clock position of the left breast roughly $8 \mathrm{~cm}$ from the nipple. There were no demonstrated mass lesions within the breast parenchyma nor any abnormal axillary lymphadenopathy.

She was referred to a surgeon who performed an incisional biopsy of the lesion under local anesthesia. Histology showed cellular proliferation of spindle cells involving the subcutaneous tissue as well as the dermis with a clear grentz zone between the overlying epidermis and the tumour. The tumour showed a predominantly storiform growth pattern with bland appearing spindle-shaped nuclei in a dense collagenous background. No pleomorphism or tumour necrosis was seen. The final tumour size was $1.5 \mathrm{~cm} \times 1.2 \mathrm{~cm}$ and the deep margin was positive for tumour. Immunohistochemical staining showed the tumour was positive for CD34 (strong and diffuse) and negative for smooth muscle actin (SMA). This picture supported the diagnosis of DFSP.

She was referred to our Women's Health Clinic for further management and was discussed at our multi-disciplinary team meeting involving the surgeons and medical oncologists. Due to her positive margins, she was counselled on the need for re-excision as well as adjuvant radiotherapy to the area. She subsequently underwent re-excision at our institution and clear margins of at least $4 \mathrm{~cm}$ were obtained. Due to the favourable location of the lesion (distant from the nippleareolar complex), breast reconstruction was not required and our patient retained breast symmetry post-operatively.

Following her surgery, she was started on imatinib. She was also offered radiotherapy but refused. She is currently 3 years disease free with no signs of recurrence. Her most recent computed tomography (CT) scan of the chest, abdomen and pelvis was normal.

\section{Discussion}

DFSP usually presents as firm, raised skin lesion with peripheral bluish discoloration. ${ }^{[9]}$ The tumour is locally invasive and can involve the lower layers of the skin in addition to the underlying muscle. As mentioned, distant metastasis is very rare but if present, usually involves the lungs. ${ }^{[10]}$

There is also a clear molecular basis for DFSB as more than 90\% demonstrate a translocation involving chromosomes 17 and 22. ${ }^{[1]}$ This results in the stimulation of platelet derived growth factor receptor (PDGFR) which consequently enhances tumour growth. A small percentage of DFSP may progress to a highly malignant and locally invasive fibrosarcoma. This is associated with a high risk of distant metastasis and carries a poor prognosis. ${ }^{[12]}$

The main differential diagnosis to consider are fibrous histiocytoma, fibrosarcoma, myxoid liposarcoma and desmoplastic melanoma. From a histological standpoint, as seen in our case, DFSP is characterised by a proliferation of bland spindle cells with a highly cellular, tight storiform architecture. ${ }^{[3]}$ If pleomorphism is present, it is usually mild. The tumour entraps adipocytes giving rise to the characteristic honeycomb pattern on microscopy (see Figure 1). Staining for CD34 demonstrates positivity in more than $90 \%$ of cases of DFSP. ${ }^{[13]}$ It is usually negative in the other tumour types mentioned and as such, carries the most weight in the definitive diagnosis of DFSP.

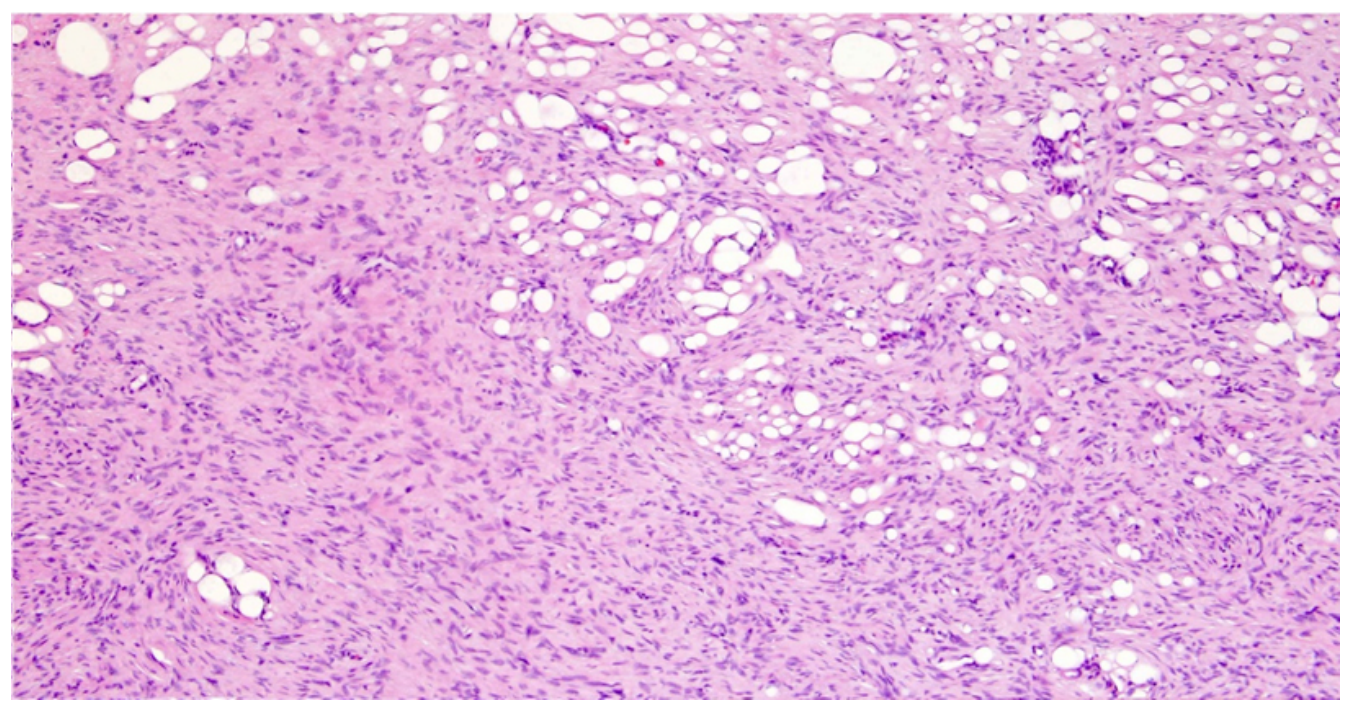

Figure 1. DFSP

Bland spindle cells with a regular storiform pattern is seen along with the honeycomb pattern on the right aspect of the slide 
There is a general consensus that the mainstay of treatment of DFSP in general is wide surgical resection. The ultimate aim is achieving clear surgical margins. Margins of $2 \mathrm{~cm}-4 \mathrm{~cm}$ has been recommended in the NCCN Guidelines. ${ }^{[8]}$ In the case of DFSP in the breast, it is important to rule out lesions within the breast parenchyma as this would alter the recommended margins of excision. Recommended margins of excision of primary breast lesions are typically less than what is required for DFSP. DFSP in the breast may warrant reconstruction given the need for wider margins. If there is doubt the adequacy of clear margins, reconstruction can be delayed. ${ }^{[8]}$ Much emphasis is made on the margins of excision as the literature has consistently shown that the recurrence rates are highest when the surgical margins are either close or positive. In fact, one study has demonstrated a $41 \%$ recurrence rate if the margins are less than $2 \mathrm{~cm}$ and an $80 \%$ five-year recurrence-free rate with margins greater than or equal to $3 \mathrm{~cm} \cdot{ }^{[14]}$

Postoperative/adjuvant targeted radiotherapy is recommended for those patients with positive or dubious margins where local re-excision may not be possible (such as unresectable or distant disease). DFSP with wide negative margins do not require adjuvant radiation. ${ }^{[5]}$ Due to the identification of the cytogenic abnormality of DFSP, medica- tions which specifically target PDGFR have been advocated. Specifically, imatinib mesylate, has been FDA approved for the treatment of DFSP with positive margins in patients who are not candidates for re-excision or those with metastatic DFSP. ${ }^{[5]}$ However, many other studies have advocated its use in the adjuvant setting in all patients with DFSP. ${ }^{[15]}$

\section{Conclusions}

DFSP is a rare skin tumour and very few cases have been reported in the breast. Surgical resection with wide margins is the gold-standard of treatment in these patients. If these lesions are not differentiated from intraparenchymal breast lesions, then the surgical resections margins would be inadequate and therefore accurate interpretation of breast imaging is very important. Re-excision is best avoided by considering the diagnosis of DFSP which warrants a wider excision at the outset. Radiotherapy and imatinib may be utilised in the adjuvant setting.

Due to the high rates of local recurrence for DFSP, regular short-term follow-up is recommended. Any suspicious areas particularly involving the surgical site, should immediately be biopsied in order to detect local recurrence.

\section{CONFlicts of InTEREST Disclosure}

The authors declare they have no conflicts of interest.

\section{REFERENCES}

[1] McLoughlin PM, Girach M, Wood GA. Dermatofibrosarcoma protuberans of the scalp. British Journal of Oral and Maxillofacial Surgery. 1992; 30: 401-403. https://doi .org/10.1016/0266-4356(92 ) $90211-Z$

[2] Bulliard C, Murali R, Chang LY, et al. Subcutaneous dermatofibrosarcoma protuberans in skin of the breast: may mimic a primary breast lesion. Pathology. 2007; 39(4): 446-448. PMid: 17676490. https://doi.org/10.1080/00313020701444523

[3] Bowne WB, Antonescu CR, Leung DH. Dermatofibrosarcoma protuberans: a clinicopathologic analysis of patients treated and followed at a single institution. Cancer. 2000; 88: 27112720. https://doi.org/10.1002/1097-0142(20000615)88: $12<2711$ : : AID-CNCR9>3.0.CO;2-M

[4] Kreicher KL, Kurlander DE, Gittleman HR, et al. Incidence and Survival of Primary Dermatofibrosarcoma Protuberans in the United States. Dermatological Surgery. 2016; 42 Suppl 1: S24-31. PMid: 26730971. https://doi.org/10.1097/DSS.00000000000003 00

[5] Bowne WB, Antonescu CR, Leung DH. Dermatofibrosarcoma protuberans: a clinicopathologic analysis of patients treated and followed at a single institution. Cancer. 2000; 88: 27112720. https://doi.org/10.1002/1097-0142(20000615)88: 12<2711: : AID-CNCR9>3.0.CO;2-M

[6] McLoughlin PM, Girach M, Wood GA. Dermatofibrosarcoma protuberans of the scalp. British Journal of Oral Maxillofacial Surgery.
1992; 30: 401-403. https://doi.org/10.1016/0266-4356(92 ) $90211-Z$

[7] Dragoumis DM, Katsohi LA, Amplianitis IK, et al. Late local recurrence of dermatofibrosarcoma protuberans in the skin of female breast. World Journal of Surgical Oncology. 2010; 8: 48. PMid: 20525288. https://doi.org/10.1186/1477-7819-8-48

[8] Miller SJ, Alam M, Andersen J. Dermatofibrosarcoma protuberans. Journal of the National Comprehensive Cancer Network. 2007; 5: 550-555. PMid: 17509257. https://doi.org/10.6004/jnccn. 2007.0048

[9] Wacker J, Khan-Durani B, Hartschuh W. Modified mohs micrographic surgery in the therapy of dermatofibrosarcoma protuberans: analysis of 22 patients. Annals of Surgical Oncology. 2004; 11: 438444. PMid: 15070606 . https ://doi .org/10.1245/ASO. 2004.0 6.014

[10] Dragoumis DM, Katsohi LA, Amplianitis IK, et al. Late local recurrence of dermatofibrosarcoma protuberans in the skin of female breast. World Journal of Surgical Oncology. 2010; 8: 48. PMid: 20525288. https://doi.org/10.1186/1477-7819-8-48

[11] Nishio J, Iwasaki H, Ishiguro M. Supernumerary ring chromosome in a bednar tumor (pigmented dermatofibrosarcoma protuberans) is composed of interspersed sequences from chromosomes 17 and 22: a fluorescence in situ hybridization and comparative genomic hybridization analysis. Genes Chromosomes and Cancer. 2001; 30: 305-309. https://doi.org/10.1002/1098-2264(2000)9999: 9999<: : AID-GCC1091>3.0.CD;2-R 
[12] Lemm D, Mugge LO, Mentzel T, et al. Current treatment options in dermatofibrosarcoma protuberans. Journal of Cancer Research and Clinical Oncology. 2009; 135: 653-665. PMid: 19205737. https://doi.org/10.1007/s00432-009-0550-3

[13] Mentzel T, Beham A, Katenkamp D, et al. Fibrosarcomatous ("highgrade") dermatofibrosarcoma protuberans: clinicopathologic and immunohistochemical study of a series of 41 cases with emphasis on prognostic significance. American Journal of Surgical Pathology.
1998; 22: 576-587. PMid: 9591728. https ://doi.org/10.1097/ 00000478-199805000-00009

[14] Roses DF, Valensi Q, LaTrenta G, et al. Surgical treatment of dermatofibrosarcoma protuberans. Surgery, Gynecology and Obstetrics. 1986; 162: 449-452. PMid: 3704900.

[15] Labropoulos SV, Razis ED. Imatinib in the treatment of dermatofibrosarcoma protuberans. Biologics: Targets \& Therapy. 2007; 1(4): 347-353. PMid: 19707305. 